PROCEEDINGS OF THE

AMERICAN MATHEMATICAL SOCIETY

Volume 140, Number 2, February 2012, Pages 645-650

S 0002-9939(2011)10938-9

Article electronically published on June 17, 2011

\title{
THE FAILURE OF THE FIXED POINT PROPERTY FOR UNBOUNDED SETS IN $c_{0}$
}

\author{
T. DOMÍNGUEZ BENAVIDES
}

(Communicated by Thomas Schlumprecht)

\begin{abstract}
In this paper we prove that for every unbounded convex closed set $C$ in $c_{0}$ there exists a nonexpansive mapping $T: C \rightarrow C$ which is fixed point free. This result solves in a negative sense a question that has remained open for some time in Metric Fixed Point Theory.
\end{abstract}

\section{INTRODUCTION}

A subset $C$ of a Banach space is said to satisfy the Fixed Point Property for nonexpansive mappings (FPP) if every nonexpansive self-mapping $T$ defined on $C$ has a fixed point. In the last 40 years many papers have appeared proving the existence of fixed points for nonexpansive mappings defined on bounded convex closed subsets of certain classes of Banach spaces, whereas other papers have shown the failure of the FPP for some bounded convex closed subsets of other Banach spaces. For instance, it is well known [1] that every bounded convex closed subset of a Hilbert space satisfies the FPP. Many more classes of reflexive Banach spaces share this property (the monographs [4] and 6] provide detailed information on this subject). In the nonreflexive case, one of the most relevant results was proved by B. Maurey [8], namely that every convex weakly compact subset of the sequence space $c_{0}$ satisfies the FPP. Besides this positive result, the failure of the FPP for other bounded convex closed subsets of $c_{0}$ is also well-known (see, for instance, [7). In 2004, P. Dowling et al. 3] proved the following complete characterization of the FPP for bounded convex closed subsets of $c_{0}$ : let $C$ be a bounded convex closed set in $c_{0}$. Then, $C$ satisfies the FPP if, and only if, $C$ is weakly compact. Their proof is based on the following fact, which is a consequence of Eberlein-S̆mulian and Alouglu Theorems: every bounded convex closed set in $c_{0}$, which is not weakly compact, contains a sequence which is $\sigma\left(\ell_{\infty}, \ell_{1}\right)$-convergent to a point $u \in \ell_{\infty} \backslash c_{0}$.

However, very few results have been obtained whenever the boundedness assumption is removed. The problem is only completely solved in the case of Hilbert spaces, because W. Ray [10] has proved that every unbounded convex subset of a Hilbert space fails to satisfy the FPP. The proof is strongly based upon the structure of Hilbert spaces, nominally, on the existence of an orthogonal basis with some specific properties. A much simpler proof of the same result has been given by R. Sine

Received by the editors November 9, 2010 and, in revised form, December 3, 2010.

2010 Mathematics Subject Classification. Primary 47H09, 47H10; Secondary 46B20.

The author was partially supported by MCIN, Grant MTM 2009-10696-C02-01, and Andalusian Regional Government Grant FQM-127. 
[13, but his proof is still based upon a property that, for dimension greater than 2 , is a characteristic of Hilbert spaces, namely that every convex closed subset of a Hilbert space is a nonexpansive retraction of the whole space. Due to this strong dependence on some characteristic properties of Hilbert spaces, it was conjectured ([5], Question XIV) that the failure of the Fixed Point Property (FPP) for every unbounded convex set could be a characterization of Hilbert spaces.

On the other hand, this problem is completely solved if you consider the Approximate Fixed Point Property (AFPP) instead of the FPP. Recall that $C$ is said to satisfy the AFPP if for every nonexpansive mapping $T: C \rightarrow C$ we have that $\inf \{\|x-T x\|: x \in C\}=0$. S. Reich [11 proved the following characterization of the sets satisfying the AFPP in a reflexive space: let $C$ be a convex closed subset of a reflexive Banach space $X$. Then, $C$ satisfies the AFPP if, and only if, $C$ is linearly bounded, that is, $C \cap r$ is bounded for every line $r$ in $X$. (A previous result for certain classes of reflexive Banach spaces was given in [9.) I. Shafrir [12] defined the notion of directional boundedness (which is equivalent to linear boundedness in reflexive spaces) and extended the above result to arbitrary Banach spaces in the following sense: let $C$ be a convex closed subset of a Banach space $X$. Then, $C$ satisfies the AFPP if, and only if, $C$ is directionally bounded.

In our paper we will study the failure of the FPP for unbounded convex sets of $c_{0}$. Since a set which fails to satisfy the AFPP also fails to satisfy the FPP, Shafrir's result will be very important in our approach. We will show that every unbounded convex directionally bounded set in $c_{0}$ contains a sequence which is $\sigma\left(\ell_{\infty}, \ell_{1}\right)$-convergent to a point $u \in \ell_{\infty} \backslash c_{0}$. As a consequence of this fact, we can adapt the proof in [3] to the unbounded case and we can prove that every unbounded convex closed set in $c_{0}$ fails to satisfy the FPP. Hence, we can respond negatively to the question raised by A. Kirk [5], proving that the failure of the FPP for every unbounded convex closed set is not a characteristic of Hilbert spaces.

\section{Preliminaries}

In [12] the notion of a directionally bounded set is stated for any hyperbolic metric space. Since we are only interested in the case of a Banach space, we will use the following equivalent definition (see [12, Theorems 2.4 and 3.2 for the equivalence):

Definition 2.1. A convex subset $C$ of a Banach space $X$ is directionally bounded if for every sequence $\left(x_{n}\right)$ in $C$ such that $\left\|x_{n}\right\| \rightarrow \infty$ and every $f \in X^{*},\|f\|=1$, one has

$$
\limsup _{n \rightarrow \infty} f\left(\frac{x_{n}}{\left\|x_{n}\right\|}\right)<1 \text {. }
$$

The following simple consequence of the above definition will be a basic tool in this paper:

Lemma 2.2. Let $C$ be a directionally bounded set in $c_{0}$ and $\left(x_{n}\right)$ be a sequence in $C$ such that $\left\|x_{n}\right\| \rightarrow \infty$. Then, for every pair of positive integers $n_{0}, k_{0}$, there exist $n>n_{0}$ and $k>k_{0}$ such that $\left|x_{n}(k)\right|=\left\|x_{n}\right\|$.

Proof. Otherwise $\left\|x_{n}\right\|=\left|x_{n}(k)\right|$ for every $n>n_{0}$ and some $k=k(n) \leq k_{0}$. Thus, there exists a subsequence $\left(x_{n_{j}}\right)$ and $\bar{k} \leq k_{0}$ such that $\pm x_{n_{j}}(\bar{k})=\left\|x_{n_{j}}\right\|$ for every 
$j \in \mathbb{N}$. Hence

$$
\limsup _{n \rightarrow \infty} \frac{x_{n}(\bar{k})}{\left\|x_{n}\right\|}= \pm 1
$$

which is a contradiction according to Definition 2.1 because the mapping $x \rightarrow x(k)$ is a normalized functional in $c_{0}^{*}$.

\section{MAIN RESUlts}

Lemma 3.1. Let $C$ be a directionally bounded convex set in $c_{0}$ and $\left(x_{n}\right)$ a sequence in $C$ such that $\left\|x_{n}\right\| \rightarrow \infty$. Denote

$$
\lambda(k)=\limsup _{n \rightarrow \infty} \frac{x_{n}(k)}{\left\|x_{n}\right\|}
$$

and by $\lambda$ the sequence $(\lambda(k))_{k} \in \ell_{\infty}$. Assume that $\lambda \neq 0$. Then, $\|\lambda\|>|\lambda(k)|$ for every $k \in \mathbb{N}$. In particular, $\lambda \notin c_{0}$.

Proof. By contradiction, we assume that there exists $k_{1}$ such that $a=:\|\lambda\|=$ $\left|\lambda\left(k_{1}\right)\right|>0$. Without loss of generality we can assume that $0 \in C$. Taking a subsequence we can assume that $x_{n}\left(k_{1}\right)$ has constant sign for every $n \in \mathbb{N}$,

$$
\left\|x_{n}\right\| \geq 2^{n}
$$

and

$$
\frac{\left|x_{n}\left(k_{1}\right)\right|}{\left\|x_{n}\right\|} \geq a\left(1-\frac{1}{2^{n}}\right)
$$

for every $n \in \mathbb{N}$. Choose $n_{1}=1$. We can choose a positive integer $k_{2}>k_{1}$ such that $\left|x_{n_{1}}(k)\right|<a / 2$ if $k \geq k_{2}$ and another integer $n_{2}>n_{1}$ such that

$$
\frac{\left|x_{n_{2}}(k)\right|}{\left\|x_{n_{2}}\right\|}<a\left(1+\frac{1}{2^{2}}\right)
$$

if $k_{1} \leq k \leq k_{2}$. Following an induction argument we find two increasing sequences $\left(n_{j}\right),\left(k_{j}\right)$ of positive integers such that

$$
\begin{gathered}
\left|x_{n_{j}}(k)\right|<\frac{a}{2^{j}} \text { if } k \geq k_{j+1}, \\
\frac{\left|x_{n_{j}}(k)\right|}{\left\|x_{n_{j}}\right\|}<a\left(1+\frac{1}{2^{j}}\right) \text { if } k_{1} \leq k \leq k_{j} .
\end{gathered}
$$

Define

$$
u_{m}=\sum_{i=1}^{m} \frac{x_{n_{i}}}{\left\|x_{n_{i}}\right\|} .
$$

It is clear from (3.1) that $u_{m}$ belongs to $C$. Furthermore, from (3.2)

$$
\left|u_{m}\left(k_{1}\right)\right| \geq(m-1) a,
$$

which, in particular, implies $(m-1) a \leq\left\|u_{m}\right\| \rightarrow \infty$.

Choose $j_{0}>4 / a$. If $k \geq k_{j_{0}-1}$, there exists $j \geq j_{0}$ such that $k_{j-1} \leq k<k_{j}$. The inequality $k<k_{j}$ and (3.4) imply

$$
\frac{\left|x_{n_{i}}(k)\right|}{\left\|x_{n_{i}}\right\|}<a\left(1+\frac{1}{2^{i}}\right)
$$

for every $i \geq j$. For $i<j-1$, we have

$$
\left|x_{n_{i}}(k)\right|<\frac{a}{2^{i}}
$$


from (3.3), because $k \geq k_{j-1} \geq k_{i+1}$. Hence, for $m \geq j$, we have

$$
\begin{aligned}
\left|u_{m}(k)\right| & \leq \sum_{i=1}^{m} \frac{\left|x_{n_{i}}(k)\right|}{\left\|x_{n_{i}}\right\|} \leq a \sum_{i=1}^{j-2} \frac{1}{2^{i}}+1+a \sum_{i=j}^{m}\left(1+\frac{1}{2^{i}}\right) \\
& \leq a+1+(m-j+1) a \leq a+1+\left(m-j_{0}+1\right) a \\
& <(m-1) a \leq\left\|u_{m}\right\| .
\end{aligned}
$$

For $j_{0} \leq m<j$ we have

$$
\begin{aligned}
\left|u_{m}(k)\right| & \leq a \sum_{i=1}^{j-2} \frac{1}{2^{i}}+1<a+1 \leq a+1+\left(m-j_{0}+1\right) a \\
& <(m-1) a \leq\left\|u_{m}\right\| .
\end{aligned}
$$

Thus, $\left|u_{m}(k)\right|<\left\|u_{m}\right\|$ for every $k \geq k_{j_{0}-1}$ and $m \geq j_{0}$, which is a contradiction according to Lemma 2.2

Lemma 3.2. Let $C$ be an unbounded convex set in $c_{0}$ which is directionally bounded. Then, there exists a sequence in $C$ which is $\sigma\left(\ell_{\infty}, \ell_{1}\right)$-convergent to a point $u \in$ $\ell_{\infty} \backslash c_{0}$.

Proof. Choose a sequence $\left(x_{n}\right)$ in $C$ such that $\left\|x_{n}\right\| \rightarrow \infty$. Without loss of generality we can assume that $0 \in C$, and taking subsequences and using a diagonal argument we can assume that $\lambda(k)=\lim _{n \rightarrow \infty} x_{n}(k) /\left\|x_{n}\right\|$ does exist for every $k \in \mathbb{N}$. If $(\lambda(k)) \in \ell_{\infty} \backslash c_{0}$ we can finish the proof by choosing the sequence $\left(x_{n} /\left\|x_{n}\right\|\right)$. Otherwise, by Lemma 3.1. $\lambda(k)=0$ for every $k \in \mathbb{N}$. We can assume that $\left\|x_{n}\right\| \geq 2^{n}$. By using Lemma 2.2 and the gliding hump method we can construct two increasing sequences $k_{j}$ and $n_{j}$ of positive integers such that:

(i) $\left|x_{n_{j}}\left(k_{j}\right)\right|=\left\|x_{n_{j}}\right\|$.

(ii) $\frac{\left|x_{n_{j}}(k)\right|}{\left\|x_{n_{j}}\right\|}<2^{-(j+1)}$ if $k \leq k_{j-1}$.

(iii) $\left|x_{n_{j-1}}(k)\right|<2^{-j}$ if $k \geq k_{j}$.

Indeed, choose $n_{1}=1$ and $k_{1} \in \mathbb{N}$ such that $\left\|x_{n_{1}}\right\|=\left|x_{n_{1}}\left(k_{1}\right)\right|$. Assume that $k_{j}, n_{j}$ have been chosen satisfying (i), (ii) and (iii) for $j \leq i-1$. We can find a positive integer $k_{i}$, large enough, such that $\left|x_{n_{i-1}}(k)\right|<2^{-i}$ if $k \geq k_{i}$ and another positive integer $n_{i}$, also large enough, such that $\left|x_{n}(k)\right| /\left\|x_{n}\right\|<2^{-(i+1)}$ if $k \leq k_{i-1}$ and $n \geq n_{i}$. From Lemma 2.2 we can also assume that $\left|x_{n_{i}}\left(k_{i}\right)\right|=\left\|x_{n_{i}}\right\|$, and so (i), (ii) and (iii) are satisfied for every $j \in \mathbb{N}$. Define the sequence

$$
v_{m}=\sum_{i=1}^{m} \frac{x_{n_{i}}}{\left\|x_{n_{i}}\right\|} .
$$

We claim that $\left(v_{m}\right)$ is a bounded sequence. Indeed, for any $k \in \mathbb{N}$ there exists $j \in \mathbb{N}$ such that $k_{j-1} \leq k<k_{j}$, where we assume $k_{0}=1$. Thus, from (ii) and (iii)

$$
\frac{\left|x_{n_{i}}(k)\right|}{\left\|x_{n_{i}}\right\|}<\frac{1}{2^{i+1}}
$$


if $i \neq j-1, j$. Hence

$$
\left|v_{m}(k)\right| \leq 2+\sum_{i=1 ; i \neq j, j-1}^{m} \frac{1}{2^{i+1}}<3 .
$$

On the other hand, for $m>j$ we have

$$
\left|v_{m}\left(k_{j}\right)\right| \geq \frac{\left|x_{n_{j}}\left(k_{j}\right)\right|}{\left\|x_{n_{j}}\right\|}-\sum_{i=1, i \neq j}^{m} \frac{1}{2^{i+1}} \geq \frac{1}{2} .
$$

By Alaoglu's Theorem, $\left(v_{m}\right)$ has a subsequence which is $\sigma\left(\ell_{\infty}, \ell_{1}\right)$-convergent to a vector $u \in \ell_{\infty} \backslash c_{0}$.

Theorem 3.3. Let $C$ be a convex closed subset of $c_{0}$. Then, $C$ satisfies the FPP for nonexpansive mappings if and only if $C$ is weakly compact.

Proof. It is well-known [8] that $C$ satisfies the FPP if it is weakly compact. To prove the converse, note that if $C$ is a bounded convex set which is not not weakly compact, then $C$ fails to satisfy the FPP [3]. Furthermore, if $C$ is convex and unbounded, then $C$ fails to satisfy the AFPP, and so the FPP, whenever $C$ is not directionally bounded. Therefore, we only need to prove that $C$ fails to satisfy the FPP if it is an unbounded closed convex set which is directionally bounded. In this case, by Lemma 3.2, $C$ contains a sequence which is $\sigma\left(\ell_{\infty}, \ell_{1}\right)$-convergent to a point in $\ell_{\infty} \backslash c_{0}$. Thus, $C$ contains a closed convex bounded subset $K$ which is not weakly compact, and by Theorem 4 in [2], $C$ contains a nonzero multiple of an asymptotically isometric $c_{0}$-summing basic sequence. Under this assumption, the arguments in the proof of Theorem 1 in 3 show that there is a convex closed subset $K_{0}$ of $K$ and a nonexpansive mapping $S: c_{0} \rightarrow K_{0}$ which is fixed point free. Define $T: C \rightarrow K_{0}$ to be the restriction of $S$ to $C$. Then, $T: C \rightarrow K_{0} \subset C$ is a fixed point free nonexpansive mapping.

\section{REFERENCES}

[1] F.E. Browder, Fixed point theorems for noncompact mappings in Hilbert spaces, Proc. Nat. Acad. Sci. USA 53 (1965) 1272-1276. MR0178324(31:2582)

[2] P.N. Dowling; C.J. Lennard; B. Turett, The fixed point for subsets of some classical Banach spaces, Nonlinear Analysis 49 (2002) 141-145. MR.1887917 (2002k:47115)

[3] P.N. Dowling; C.J. Lennard; B. Turett, Weak compactness is equivalent to the fixed point property in $c_{0}$, Proc. Amer. Math. Soc. 132 (2004) 1659-1666. MR2051126 (2004m:46024)

[4] K. Goebel, W.A. Kirk, Topics in Metric Fixed Point Theory. Cambridge Studies in Advanced Mathematics, 28. Cambridge University Press, Cambridge, 1990. MR.1074005 (92c:47070)

[5] W.A. Kirk, Some questions in metric fixed point theory, Recent advances on metric fixed point theory (Seville, 1995), 73-97, Ciencias, 48, Univ. Sevilla, Seville, 1996. MR 1440220 (98f:46010)

[6] Handbook of Metric Fixed Point Theory. Edited by William A. Kirk and Brailey Sims. Kluwer Academic Publishers, Dordrecht, 2001. MR1904271 (2003b:47002)

[7] E. Llorens-Fuster; B. Sims, The fixed point property in $c_{0}$, Canad. Math. Bull. 41 (1998) 413-422. MR 1658231 (99i:47097)

[8] B. Maurey, Points fixes des contractions de certains faiblement compacts de $L^{1}$, Seminar on Functional Analysis, 1980-1981, Exp. No. VIII, 19 pp., École Polytech., Palaiseau, 1981. MR659309(83h:47041)

[9] W.O. Ray, Nonexpansive mappings on unbounded convex domains, Bull. Acad. Polon. Sci. Sér. Sci. Math. Astronom. Phys. 26 (1978), no. 3, 241-245. MR0493551 (58:12545)

[10] W.O. Ray, The fixed point property and unbounded sets in Hilbert space, Trans. Amer. Math. Soc. 258 (1980) 531-537. MR558189 (81e:47044) 
[11] S. Reich, The almost fixed point property for nonexpansive mappings, Proc. Amer. Math. Soc. 88 (1983), no. 1, 44-46. MR691276 (84g:47052)

[12] I. Shafrir, The approximate fixed point property in Banach and hyperbolic spaces, Israel J. Math. 71 (1990), no. 2, 211-223. MR.1088815 (92b:47096)

[13] R. Sine, On the converse of the nonexpansive map fixed point theorem for Hilbert space, Proc. Amer. Math. Soc. 100 (1987), no. 3, 489-490. MR891152 (88g:47122)

Facultad de Matemáticas, Universidad de Sevilla, P.O. Box 1160, 41080 Sevilla, Spain

E-mail address: tomasd@us.es 